\title{
Optimal Monochromatic Energy Levels in Dual-Energy Spectral CT Pulmonary Angiography with Low Contrast Medium Dosage
}

\author{
Dong $\operatorname{Han}^{1,2}$, Weihua Shi ${ }^{1}$, Xiaoxia Chen ${ }^{1}$, Jieli Zhou ${ }^{3}$, Yong Yu ${ }^{1,2}$, Xin Tian $^{1}$, Jing Chen ${ }^{1}$, Mengting Liu ${ }^{2}$ \\ and Taiping $\mathrm{He}^{1,2,{ }^{*}}$
}

${ }^{1}$ Department of Radiology, Affiliated Hospital of Shaanxi University of Traditional Chinese Medicine, Xianyang, China

${ }^{2}$ College of Medical Technology, Shaanxi University of Traditional Chinese Medicine, Xianyang, China

${ }^{3}$ Department of Ultrasound, Xijing Hospital, Air Force Medical University, Xi'an, China

"Corresponding author: Department of Radiology, Affiliated Hospital of Shaanxi, University of Traditional Chinese Medicine, Xianyang, China. Email: 523785125@qq.com

Received 2018 August 20; Revised 2020 February 05; Accepted 2020 February 18.

\begin{abstract}
Background: The contrast medium (CM) in CT pulmonary angiography may induce adverse effects to patients, and higher CM is associated with higher rates of contrast-induced-nephropathy and mortality. Reduction of CM dosage through improvement of examination techniques may help reduce the occurrence of CM-induced adverse effects and healthcare costs.

Objectives: To determine the optimal monochromatic energy levels in dual-energy spectral CT pulmonary angiography (CTPA) with low contrast dosage.

Patients and Methods: Thirty patients with suspected pulmonary embolism (PE) underwent dual-energy spectral CTPA with low radiation and low contrast doses with scanning protocol of GSI-36 with $260 \mathrm{~mA}$, and $25 \mathrm{~mL}$ contrast (350 mgI/mL) with $4.0 \mathrm{ml} / \mathrm{s}$ injection speed. The monochromatic images from $60-80 \mathrm{keV}$ (interval $5 \mathrm{keV}$ ) were reconstructed using a 50\% adaptive statistical iterative reconstruction (50\% ASiR) algorithm at $1.25 \mathrm{~mm}$ slice thickness. The CT attenuation and standard deviation (SD) values of the main, right, left, right lower and left lower pulmonary arteries and the back muscle at the same level were measured on $60-80$ keV images, and signal-to-noise ratio (SNR) and contrast-to-noise ratio (CNR) were calculated and analyzed. The subjective image quality was evaluated by two experienced radiologists using a 5-level scoring method independently. Measurements were analyzed using IBM SPSS Statistics for Windows, version 25.0. (Armonk, NY: IBM Corp.).

Results: CT attenuation values of the pulmonary arteries decreased with the increase of energy level in five-energy groups, with values greater than $300 \mathrm{HU}$ at $60 \mathrm{keV}-70 \mathrm{keV}$ energies. The $65 \mathrm{keV}$ image had the highest SNR, CNR and lowest SD, with significant differences compared with those of other image sets $(\mathrm{P}<0.05)$. The subjective quality scores for the $65 \mathrm{keV}$ image was judged to be the highest by the two radiologists, but it was not significantly different from $60 \mathrm{keV}$ and $70 \mathrm{keV}$ (all $\mathrm{P}>0.05$ ).

Conclusion: The $65 \mathrm{keV}$ monochromatic images provided the highest SNR, CNR and subjective scores with the lowest image noise in dual-energy spectral CTPA with low contrast dosage.
\end{abstract}

Keywords: Low Contrast Medium Dosage, CT Pulmonary Angiography, Spectral CT, Optimal Monochromatic Energy Level

\section{Background}

Pulmonary embolism (PE) is an obstruction in pulmonary arteries and their branches. It ranks third in the most common cardiovascular diseases with about 100 200 in 10 million in morbidity (1). Pulmonary angiography has been regarded as the gold standard for the diagnosis or exclusion of pulmonary embolism for decades, but non-invasive CT pulmonary angiography (CTPA) provides similar diagnostic accuracy. According to the PIOPED II (prospective study of PE diagnosis) test, the sensitivity was $83 \%$ and the specificity was $96 \%$ for CTPA with multi-slice CT (MSCT) (1). The observer confidence was up to $100 \%$ for
PE diagnosis (2). CTPA has become the preferred method in the diagnosis or exclusion of patients with suspected pulmonary embolism (3-5).

Contrast medium (CM) is required in CTPA, and the CM may cause negative effects for the patients, among which contrast-induced nephropathy (CIN) is particularly prominent (6). If concurrent with diabetes and renal insufficiency, the morbidity of CIN could reach $19 \%$ or more (7), which is associated with mortality of patients and the third primary cause of hospital acquired renal failure (8). CM is almost completely excreted by the kidneys; therefore, higher contrast volume is associated with higher rates of 
CIN and mortality (9). The key is to reduce the occurrence of CM-induced adverse effects and healthcare costs by improving examination techniques to reduce $\mathrm{CM}$ dosage (10).

However, reducing CM dosage will have a negative impact on the pulmonary artery enhancement in conventional CTPA, and may impair the diagnostic tasks. Therefore, CM should be used as least as possible under the condition of meeting diagnostic requirements (11). On the other hand, imaging techniques have been improved constantly to provide better images with less radiation and contrast doses. The recently introduced dual-energy spectral CT provides monochromatic images with energy levels from $40 \mathrm{keV}$ to $140 \mathrm{keV}$ that could be used to improve the contrast-noise-ratio (CNR) in target vessels. CT values of the blood vessel in dual-energy spectral CTPA were significantly increased at low energy levels to provide the possibility of using low CM dosage in CTPA. However, the monochromatic energy levels in dual-energy spectral CTPA used by researchers were not consistent $(2,5,7)$.

\section{Objectives}

The purpose of this study was to investigate the feasibility of using low CM volume in dual-energy spectral CTPA, and to determine the optimal monochromatic energy levels of displaying pulmonary arteries by quantitively and qualitatively analyzing the images with low $\mathrm{CM}$ volume, under the premise of ensuring diagnosis of PE.

\section{Patients and Methods}

\subsection{Patients Group}

This single-site prospective study was approved by our Institutional Review Board (the approval document no.: SZFYIEC-PF-2015 no. [08]), and written informed consents were received from all patients before CTPA examination. Thirty patients who were suspected of PE and underwent CTPA were collected from November 2015 to May 2016, 17 were males and 13 were females, the average age was 67.40 \pm 9.15 years. Exclusion criteria were allergy to iodine CM, hyperthyroidism, severe liver and kidney dysfunction, severe non-compensatory cardiac insufficiency, pregnancy, and less than 18 years of age.

\subsection{Data Acquisition and Reconstruction}

All examinations were performed on a 64-slices dualenergy spectral CT scanner (discovery CT 750 HD, GE Healthcare, USA). Patients were trained to hold their breath after inspiration before the examination. Patients were scanned in supine position and craniocaudal direction, with both forearms raised to the head. The scanning range was from the apex to the costophrenic angle and completed while holding breath. The dual-energy spectral CT scanning protocol: GSI-36 (with tube current of $260 \mathrm{~mA}$ ) was chosen. Scanning and reconstruction parameters are shown in Table 1 . The scanning delay time was determined by using the timing bolus technique with the region of interest (ROI) placed in the pulmonary trunk at the aortic arch level. A $8 \mathrm{~mL}$ non-ionic iodinated CM (Ioversol, 350 mgI/mL; Jiangsu Hengrui Medicine Co. Ltd., China) was injected at a rate of $4.0 \mathrm{~mL} / \mathrm{s}$ via the antecubital vein using a high-pressure syringe (Missouri XD2001, Ulrich medical, Buchbrunnenweg, Ulm, Germany) for test bolus (TB). The dynamic monitoring scanning, each monitoring point interval was set at 1 s to generate the time-density curve, and to determine the best delay time from injection to peak enhancement. Subsequently, $25 \mathrm{~mL}$ CM of the same concentration was injected at the same rate and the scan started using the determined delay time +2 seconds for CTPA data acquisition. After the $\mathrm{CM}$ injection, $40 \mathrm{ml}$ of normal saline was injected. After scanning, monochromatic images with photon energies of $60-80 \mathrm{keV}$ (at energy incremental of $5 \mathrm{keV}$ ) using the $50 \%$ adaptive statistical iterative reconstruction (50\% ASiR) algorithm were retrospectively reconstructed and transferred to AW4.6 post processing workstation (GE Healthcare) for analysis.

\begin{tabular}{lc}
\hline $\begin{array}{l}\text { Table 1. Scanning and Reconstruction Parameters of Dual-Energy Spectral CTPA at } \\
\text { Low Contrast Dose }\end{array}$ \\
\hline Scanning parameters & Values \\
\hline Scanning protocol & GSI-36: $260 \mathrm{~mA}$ \\
\hline Pitch & $1.375: 1$ \\
\hline Rotation rate & $0.8 \mathrm{~s} / \mathrm{r}$ \\
\hline CM volume & $25 \mathrm{~mL}(350 \mathrm{mgI} / \mathrm{mL}, 4.0 \mathrm{~mL} / \mathrm{s})$ \\
\hline Monitoring method & timing bolus (8 mL) \\
\hline Reconstruction parameters & $1.25 \mathrm{~mm}$ \\
\hline Thickness & $50 \%$ ASiR \\
\hline Algorithm & Virtual monochromatic images with $60-80$ \\
Reconstructed images & keV (5 keV incremental) \\
\hline Abbreviations: CM, contrast medium; CTPA, CT pulmonary angiography.
\end{tabular}

\subsection{Data Measurement and Calculation}

\subsubsection{Objective Parameters}

The region-of-interest (ROI) was placed in the lumen of the main pulmonary artery (MPA), the right pulmonary artery (RPA), the left pulmonary artery (LPA), the right 
lower pulmonary artery (RLPA) and the left lower pulmonary artery (LLPA) as well as the back muscle at the same level of the measured pulmonary artery on the axial sectional images of the five energy levels in each patient by a radiologist with 8 years of experience in cardiovascular diagnosis. CT values and standard deviation (SD), which was used as the noise of images in the ROI were measured. The edge of blood vessels and embolus in vessels were avoided when placing ROI, and measurements were carried out three times on three consecutive levels to obtain an average value, shown in Figure 1.

The signal-to-noise ratio (SNR) and CNR of each pulmonary artery was calculated using the following formulas:

SNR = CT value (pulmonary artery)/SD (pulmonary artery)

$\mathrm{CNR}=(\mathrm{CT}$ value (pulmonary artery) $-\mathrm{CT}$ value (back muscle))/SD (back muscle)

The volumetric CT dose index $\left(\mathrm{CTDI}_{\mathrm{vol}}\right)$ and dose length product (DLP) of each patient were recorded in the dose report, and the effective dose (ED) was calculated using the following formula:

$\mathrm{ED}=\mathrm{DLP} \times \mathrm{K}(\mathrm{K}=0.014)(12)$

\subsubsection{Subjective Evaluation}

Image quality was evaluated by two experienced radiologists using blind method and 5-point scoring system for the five image groups independently. The two radiologists were not informed about the scan methods of these patients, and the image parameters were not displayed before evaluation. The evaluation criteria are shown in Table 2.

\begin{tabular}{ll}
\hline \multicolumn{2}{l}{ Table 2. Subjective Evaluation Criteria of CTPA Image Quality } \\
\hline Score level & \multicolumn{1}{c}{ Evaluation criterion } \\
\hline $\mathbf{5}$ & $\begin{array}{l}\text { Excellent image quality, no obvious noise and artifacts, fully } \\
\text { diagnostic. }\end{array}$ \\
$\mathbf{4}$ & Good image quality, minor noise and artifacts, fully diagnostic. \\
$\mathbf{3}$ & $\begin{array}{l}\text { Limited image quality, moderate noise and artifacts, still } \\
\text { diagnostic. }\end{array}$ \\
$\mathbf{2}$ & $\begin{array}{l}\text { Suboptimal image quality, obvious noise and artifacts, } \\
\text { affecting the diagnosis. }\end{array}$ \\
\hline $\mathbf{1}$ & $\begin{array}{l}\text { Poor image quality, high noise and severe artifacts, } \\
\text { non-diagnostic. }\end{array}$ \\
\hline Abbreviation: CTPA, CT pulmonary angiography.
\end{tabular}

Abbreviation: CTPA, CT pulmonary angiography.

\subsection{Statistical Analysis}

The measurements were analyzed by using IBM SPSS ${ }^{\circledR}$ statistics version 25.0. (New York, IBM Corp.), with $\mathrm{P}<0.05$ indicating statistically significant difference. Continuous variables were expressed as mean \pm standard deviation, the subjective score was expressed as median (Q1, Q3). The single sample K-S and Levene's tests were used to test the goodness of fit and homogeneity of variance of the measurements respectively. The one-way repeated measure ANOVA was used to test the parameters of the five-energy level monochromatic image groups which were consistent with normal distribution and homogeneity of variance, and the parameters that do not satisfy Mauchly's spherical test were corrected by Greenhouse-Geisser method. The intraclass correlation efficient (ICC) was used to test the repeatability of quantitative data measurements. WeightedKappa test was used to evaluate the consistency of subjective scores by the two radiologists, the Kappa or ICC values were defined as follows: > 0.75, excellent agreement; 0.60 - 0.74, good agreement; 0.40 - 0.59, fair agreement; and $<0.40$, poor agreement. The subjective scores of the 5-energy level groups were compared by Friedman M nonparametric test. Cochran's Q test was used to compare the frequency of subjective scores greater than 3 in five groups. Correlation analysis was performed by using Pearson or Spearman correlation analysis. Bonferroni method was used to calibrate all variables in the two comparisons.

\section{Results}

\subsection{General Information of Patients and Effective Dose}

The average height, weight and body mass index (BMI) of the 30 patients was $1.66 \pm 0.08 \mathrm{~m}, 62.87 \pm 10.52 \mathrm{~kg}$ and $22.93 \pm 3.79 \mathrm{~kg} . \mathrm{m}^{-2}$, respectively. The average $\mathrm{CTDI}_{\mathrm{vol}}$, DLP and ED was $9.12 \pm 3.03 \mathrm{mGy}, 264.65 \pm 74.81 \mathrm{mGy} . \mathrm{cm}$, and $3.70 \pm 1.05 \mathrm{mSv}$, respectively.

\subsection{Objective Parameters of the Monochromatic Images}

4.2.1. Comparison of CT Value of Different Energy Levels in Pulmonary Arteries

The ICC of the three measurements of pulmonary arteries was $0.985 \pm 0.009$, all $\mathrm{P}<0.001$. The CT attenuation values of the pulmonary arteries decreased with the increase of energy level in the five monochromatic image groups from $60 \mathrm{keV}$ to $80 \mathrm{keV}$, as shown in Figure 2. The mean CT attenuation values of pulmonary arteries at these five energy levels were $431.41 \pm 129.04$ hounsfield unit (HU), $372.59 \pm 112.42 \mathrm{HU}, 319.26 \pm 92.67 \mathrm{HU}, 265.96 \pm 75.34 \mathrm{HU}$ and $232.87 \pm 65.34 \mathrm{HU}$, respectively. The difference was statistically significant $(\mathrm{F}=180.62, \mathrm{P}<0.001)$. Among them, the CT attenuation values of $60 \mathrm{keV}, 65 \mathrm{keV}$, and $70 \mathrm{keV}$ images were higher than $300 \mathrm{HU}$, as shown in Table 3. 

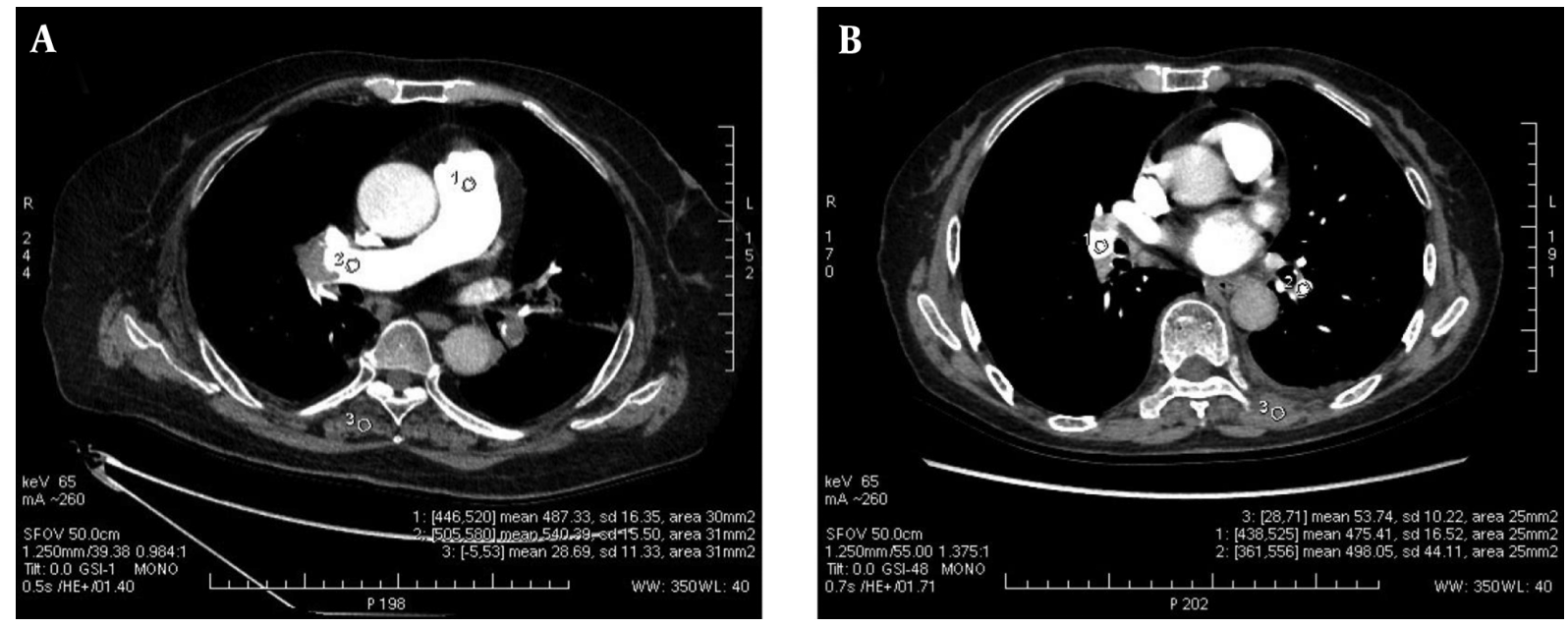

Figure 1. Example of region of interest (ROI) placement in CT pulmonary angiography (CTPA) images

\begin{tabular}{|c|c|c|c|c|c|c|c|}
\hline Pulmonary arteries & $60 \mathrm{keV}$ & $65 \mathrm{keV}$ & $70 \mathrm{keV}$ & $75 \mathrm{keV}$ & $80 \mathrm{keV}$ & $\mathbf{F}$ & $\mathbf{P}$ \\
\hline MPA, HU & $401.66 \pm 133.16$ & $346.60 \pm 118.21$ & $298.69 \pm 97.55$ & $240.61 \pm 90.21$ & $213.24 \pm 70.82$ & 145.90 & $<0.001$ \\
\hline RPA, HU & $442.34 \pm 139.11$ & $390.83 \pm 120.59$ & $327.68 \pm 94.01$ & $281.94 \pm 79.97$ & $246.37 \pm 67.15$ & 128.29 & $<0.001$ \\
\hline LPA, HU & $438.49 \pm 142.17$ & $380.89 \pm 120.41$ & $332.51 \pm 101.63$ & $273.39 \pm 76.28$ & $239.04 \pm 66.95$ & 118.01 & $<0.001$ \\
\hline RLPA, HU & $432.45 \pm 129.50$ & $367.17 \pm 117.54$ & $307.76 \pm 94.63$ & $259.62 \pm 74.38$ & $225.16 \pm 73.76$ & 147.11 & $<0.001$ \\
\hline LLPA, HU & $442.12 \pm 122.13$ & $377.48 \pm 107.04$ & $329.64 \pm 88.70$ & $274.25 \pm 74.89$ & $240.54 \pm 66.30$ & 197.95 & $<0.001$ \\
\hline Mean, HU & $431.41 \pm 129.04$ & $372.59 \pm 112.42$ & $319.26 \pm 92.67$ & $265.96 \pm 75.34$ & $232.87 \pm 65.34$ & 180.62 & $<0.001$ \\
\hline
\end{tabular}

Abbreviations: HU, Hounsfield unit; LLPA, left lower pulmonary artery; LPA, left pulmonary artery; MPA, main pulmonary artery; RLPA, right lower pulmonary artery; RPA, right pulmonary artery; SD, standard deviation.

${ }^{a}$ Values are expressed as mean $\pm S D$.

${ }^{\mathrm{b}} 65 \mathrm{keV}$ vs. other keV (adjusted by Bonferroni test): all adjust-P $<0.001$ [all differences between $65 \mathrm{keV}$ vs. $60,70,75$, and 80 keV were statistically significant; $\mathrm{P}<0.001$ ].

\subsubsection{Comparison of SNR, CNR, and SD}

ICC of the three measurements of image noise was $0.847 \pm 0.136$ (all $\mathrm{P}<0.001$ ).

In the five monochromatic image groups from $60 \mathrm{keV}$ to $80 \mathrm{keV}$, the mean SNR values were $22.02 \pm 7.04 \mathrm{HU}, 27.57$ $\pm 10.05 \mathrm{HU}, 18.52 \pm 5.96 \mathrm{HU}, 16.21 \pm 5.03 \mathrm{HU}$, and $14.43 \pm$ $4.94 \mathrm{HU}$ respectively, and the differences were statistically significant $(\mathrm{F}=62.93, \mathrm{P}<0.001)$. The mean $\mathrm{CNR}$ values were $24.79 \pm 11.08 \mathrm{HU}, 32.97 \pm 14.07 \mathrm{HU}, 20.41 \pm 8.13 \mathrm{HU}, 15.63$ $\pm 5.56 \mathrm{HU}$, and $12.88 \pm 4.81 \mathrm{HU}$, respectively, and the differences were statistically significant $(\mathrm{F}=93.07, \mathrm{P}<0.001)$. The mean SD values were $20.68 \pm 4.78,15.14 \pm 6.06,18.29$ $\pm 4.94,17.25 \pm 3.66$, and $17.03 \pm 3.82$, respectively, and the differences were also statistically significant $(\mathrm{F}=18.20, \mathrm{P}<$ 0.001).

The SNR and CNR values of the $65 \mathrm{keV}$ monochromatic image were the highest, while the SD value was the lowest among the five image groups, paired comparisons are shown in Table 4, which had statistically significant difference (all $\mathrm{P}<0.01$ ), as shown in Figures 2 and 3.

\subsection{Subjective Evaluation of Each Monochromatic Image}

Subjective evaluation of the five monochromatic groups was carried out by two radiologists. The consistency of subjective score was excellent between the two radiologists (Weighted-Kappa $=0.901, \mathrm{P}<0.001$ ), and the subjective score of the $65 \mathrm{keV}$ monochromatic image was higher than the other groups. The percentage of patients with a subjective score more than 3 points was at the highest in the $60 \mathrm{keV}$ and $65 \mathrm{keV}$ images, as shown in Figure 3, Table 5.

4.4. Correlation Between BMI with CNR and Subjective Score in the $65 \mathrm{keV}$ Monochromatic Image

There was no correlation between BMI with CNR and subjective score of the $65 \mathrm{keV}$ monochromatic images, as 


\begin{tabular}{|c|c|c|c|c|c|c|c|}
\hline Pulmonary arteries & $60 \mathrm{keV}$ & $65 \mathrm{keV}$ & $70 \mathrm{keV}$ & $75 \mathrm{keV}$ & $80 \mathrm{keV}$ & $\mathbf{F}$ & $\mathbf{P}$ \\
\hline \multicolumn{8}{|l|}{ SNR } \\
\hline MPA & $21.79 \pm 8.46^{\mathrm{b}}$ & $26.34 \pm 11.58$ & $18.50 \pm 7.59^{c}$ & $16.40 \pm 7.72^{\mathrm{c}}$ & $14.02 \pm 6.10^{c}$ & 31.99 & $<0.001$ \\
\hline RPA & $22.96 \pm 8.45^{\mathrm{d}}$ & $28.46 \pm 12.22$ & $18.29 \pm 6.28^{\mathrm{c}}$ & $16.54 \pm 5.86^{\mathrm{c}}$ & $14.78 \pm 4.44^{\mathrm{c}}$ & 38.98 & $<0.001$ \\
\hline LPA & $22.54 \pm 9.00^{d}$ & $27.51 \pm 12.27$ & $18.93 \pm 7.43^{c}$ & $16.05 \pm 5.15^{c}$ & $14.60 \pm 6.62^{c}$ & 31.04 & $<0.001$ \\
\hline RLPA & $20.39 \pm 6.38^{c}$ & $27.20 \pm 10.46$ & $18.51 \pm 6.70^{c}$ & $15.92 \pm 5.00^{c}$ & $14.24 \pm 5.97^{\mathrm{c}}$ & 40.73 & $<0.001$ \\
\hline LLPA & $22.43 \pm 7.44^{\mathrm{d}}$ & $28.35 \pm 11.13$ & $18.37 \pm 5.65^{c}$ & $16.12 \pm 5.64^{c}$ & $14.51 \pm 4.37^{c}$ & 41.48 & $<0.001$ \\
\hline Mean & $22.02 \pm 7.04^{\mathrm{d}}$ & $27.57 \pm 10.05$ & $18.52 \pm 5.96^{c}$ & $16.21 \pm 5.03^{\mathrm{c}}$ & $14.43 \pm 4.94^{c}$ & 62.93 & $<0.001$ \\
\hline \multicolumn{8}{|l|}{ CNR } \\
\hline MPA & $23.11 \pm 10.41^{\mathrm{C}}$ & $29.36 \pm 12.48$ & $18.22 \pm 7.80^{c}$ & $13.53 \pm 6.25^{\mathrm{c}}$ & $10.72 \pm 3.94^{\mathrm{c}}$ & 95.04 & $<0.001$ \\
\hline RPA & $26.48 \pm 12.03^{c}$ & $35.00 \pm 15.61$ & $20.93 \pm 7.73^{c}$ & $16.56 \pm 5.84^{c}$ & $13.26 \pm 4.37^{c}$ & 68.49 & $<0.001$ \\
\hline LPA & $24.58 \pm 12.45^{c}$ & $33.59 \pm 14.80$ & $21.54 \pm 9.27^{c}$ & $16.30 \pm 5.78^{c}$ & $14.28 \pm 5.66^{\mathrm{c}}$ & 55.29 & $<0.001$ \\
\hline RLPA & $24.25 \pm 11.36^{\mathrm{c}}$ & $33.23 \pm 15.37$ & $20.05 \pm 9.02^{c}$ & $15.28 \pm 5.68^{c}$ & $12.37 \pm 6.32^{c}$ & 82.37 & $<0.001$ \\
\hline LLPA & $25.55 \pm 11.96^{\mathrm{c}}$ & $33.68 \pm 15.39$ & $21.29 \pm 7.93^{c}$ & $16.46 \pm 5.97^{c}$ & $13.75 \pm 6.04^{\mathrm{c}}$ & 72.11 & $<0.001$ \\
\hline Mean & $24.79 \pm 11.08^{c}$ & $32.97 \pm 14.07$ & $20.41 \pm 8.13^{c}$ & $15.63 \pm 5.56^{c}$ & $12.88 \pm 4.81^{\mathrm{C}}$ & 93.07 & $<0.001$ \\
\hline SD, HU & $20.68 \pm 4.78^{c}$ & $15.14 \pm 6.06$ & $18.29 \pm 4.94^{c}$ & $17.25 \pm 3.66^{\mathrm{c}}$ & $17.03 \pm 3.82^{\mathrm{d}}$ & 18.20 & $<0.001$ \\
\hline
\end{tabular}

Abbreviations: CNR, contrast-to-noise ratio; HU, Hounsfield unit; LLPA, left lower pulmonary artery; LPA, left pulmonary artery; MPA, main pulmonary artery; RLPA, right lower pulmonary artery; RPA, right pulmonary artery; SNR, signal-to-noise ratio; SD, standard deviation.

${ }^{a}$ Values are expressed as mean \pm SD.

${ }^{\mathrm{b}} 65 \mathrm{keV}$ vs. other keV (adjusted by Bonferroni test) for adjust-P $>0.05$.

c $65 \mathrm{keV}$ vs. other keV (adjusted by Bonferroni test) for adjust-P $<0.001$.

${ }^{\mathrm{d}} 65 \mathrm{keV}$ vs. other keV (adjusted by Bonferroni test) for adjust-P $<0.05$.

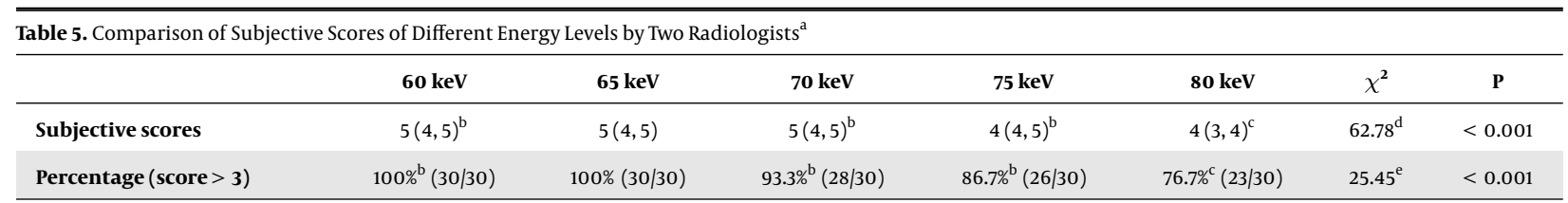

${ }^{\mathrm{a}}$ Subjective score is expressed as median $(\mathrm{Q} 1, \mathrm{Q} 3)$.

${ }^{\mathrm{b}} 65 \mathrm{keV}$ vs. other keV (adjusted by Bonferroni test): for adjust-P $>0.05$.

c $65 \mathrm{keV}$ vs. other $\mathrm{keV}$ (adjusted by Bonferroni test): a for adjust- $\mathrm{P}<0.001$.

${ }^{\mathrm{d}}$ Friedman test statistic.

${ }^{\mathrm{e}}$ Cochran's Q test statistic.

shown in Figure 4.

BMI vs. CNR (mean), Pearson- $\mathrm{R}=-0.306, \mathrm{P}=0.100$;

BMI vs. subjective scores, Spearman- $R=0.126, P=0.506$.

\section{Discussion}

The results of our study showed that under the condition of low CM volume ( $25 \mathrm{~mL}$ ), CTPA imaging using $65 \mathrm{keV}$ monochromatic images with ASiR algorithm had higher SNR, CNR and lower image noise than other energy levels, providing the best monochromatic level for diagnosing PE.

The researchers are committed to reducing the volume of $\mathrm{CM}$, the purpose of which is to reduce the iodine load of patients and reduce the adverse reaction of CM. Most institutions generally used $80-150 \mathrm{~mL}$ CM in CTPA in the past $(13,14)$. However, these CM have not been fully utilized in the contrast enhancement of the pulmonary artery. In recent years, some researchers rely on a variety of progres- sive techniques and methods, which help to optimize the CM volume $(13,15-19)$. The research conducted by Wu et al. (20) showed that the CTPA image quality and diagnostic requirements are not affected when using $30 \mathrm{~mL}$ CM. Lu et al. (21) even pushed the CM volume down to $20 \mathrm{~mL}$ when conducting CTPA researches, and showed that the diagnostic images could also be obtained in the normal weight population.

The dual-energy spectral CT imaging mode in our study uses single source and rapid switching between high and low voltages (140 kVp/80 kVp) in 0.5 milliseconds to provide coherent dual-energy information at similar radiation dose as conventional CT scan modes. The dual-energy spectral CT reconstructs virtual monochromatic images with different energy levels from $40 \mathrm{keV}$ to $140 \mathrm{keV}$, simulating the imaging under different monochromatic Xray sources $(22,23)$. Compared with conventional CT images, the virtual monochromatic images generally have 
A

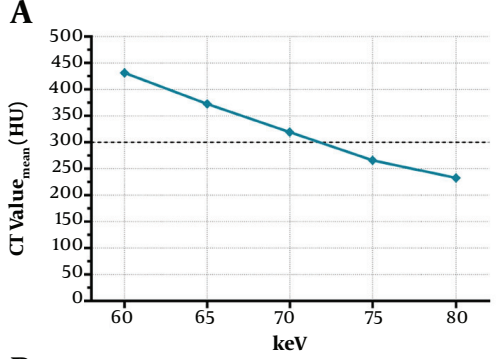

B

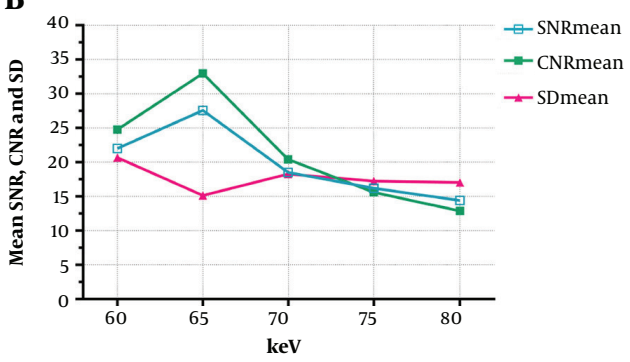

Figure 2. A, The CT attenuation values of the pulmonary arteries decreased with the increase of energy level in the five monochromatic image groups from $60 \mathrm{keV}$ to $80 \mathrm{keV}$, and the CT attenuation values of the $60 \mathrm{keV}, 65 \mathrm{keV}$, and $70 \mathrm{keV}$ images were higher than $300 \mathrm{HU}$; B, The mean SNR and CNR values of the $65 \mathrm{keV}$ monochromatic image were the highest, while the SD value was the lowest among the 5 image groups.(SNR, signal-to-noise ratio; CNR, contrast-to-noise ratio; and SD, standard deviation).

better image quality, higher $\operatorname{SNR}$ and $\operatorname{CNR}(24,25)$. The model experimental study conducted by Matsumoto et al. (26) showed that the $70 \mathrm{keV}$ monochromatic images had higher CNR and lower noise compared with the polychromatic $120 \mathrm{kVp}$ images with similar radiation dose. The monochromatic images with lower energy levels can improve the density resolution of the images, which also helps to improve the CT value of the substance being tested, but the image noise increased at the same time, while the high-energy level images have lower CT attenuation values, lower contrast ratio and lower noise. Therefore, selecting an appropriate energy level, to balance the enhancement degree and the image noise of pulmonary arteries is the key to ensure the PE diagnosis and improve the image quality.

Previous studies (27) have indicated that the optimal monochromatic energy levels of observing pulmonary thrombosis with spectral CTPA are 65-70 keV, of which the images have the lowest noise and higher CNR. The study conducted by Apfaltrer et al. (28) showed that the optimal monochromatic energy level of pulmonary arteries was $70 \mathrm{keV}$ when using dual-energy CT for CTPA examination. Ohana et al. (29), who studied the influence of iterative reconstruction algorithm on image quality, measured the quantitative parameters of pulmonary artery on
$65 \mathrm{keV}$ monochromatic images. However, the authors did not explain the reasons for using $65 \mathrm{keV}$ monochromatic images in the article. All of the above studies were under the conditions of normal CM dosage ( $80-100 \mathrm{~mL})$, and the optimal monochromatic energy level of CTPA in the diagnosis of PE was rarely reported when using low CM volume. Yuan et al. (3) in the case of iodine load decreased by $40.2 \%$, selected the $50 \mathrm{keV}$ virtual monochromatic images to measure the parameters of pulmonary arteries only by the preliminary experience and the manufacturer's recommendations, which lacked objective basis. The purpose of our study was to investigate the feasibility and the optimal monochromatic energy level in the diagnosis of PE when using dual-energy spectral CTPA with low CM volume (25 $\mathrm{mL})$.

There is no universal standard of pulmonary artery enhancement in the diagnosis of PE at present. Multiple studies $(2,3,30,31)$ showed that the minimum CT value in pulmonary arteries was $290 \pm 83 \mathrm{HU}$ and $362 \pm 98 \mathrm{HU}$ respectively in the diagnosis of acute and chronic PE. The results of our study showed that the average CT attenuation values of the pulmonary arteries were more than $300 \mathrm{HU}$ on the $60-70 \mathrm{keV}$ monochromatic images, which could meet the diagnostic requirements for $\mathrm{PE}$, among which the $65 \mathrm{keV}$ monochromatic images had the highest SNR and CNR as well as the lowest image noise. Therefore, we confirmed that the optimal monochromatic energy level in dual-energy spectral CTPA was $65 \mathrm{keV}$ when using low CM dosage.

In our study, the ASiR algorithm was used in the dualenergy spectral CTPA image reconstructions instead of the more traditional filtered back-projection reconstruction. The study conducted by Ohana et al. (29) showed that the ASiR algorithm provided good image quality in dualenergy spectral CTPA in low radiation dose condition. In our study, we used ASiR to reduce the image noise which was more prominent at the low energy levels to fully take advantage of the improved attenuation at lower energy levels to further optimize image quality.

ASiR provides $0 \%-100 \%$ weights for different clinical needs, the higher the percentage of ASiR, the lower the image noise, and the smoother the images. At high ASiR percentages the image sharpness, spatial resolution and image quality may be reduced. Previous studies have shown that the spatial resolution and image noise could be balanced at approximately 40\% - 50\% ASiR (maintain image spatial resolution while reducing noise) (32). We selected the $50 \%$ ASiR weights used by other researchers in abdominal low-dose CT scans (33) and portal vein imaging studies $(34,35)$. This was also the most commonly used weight in 

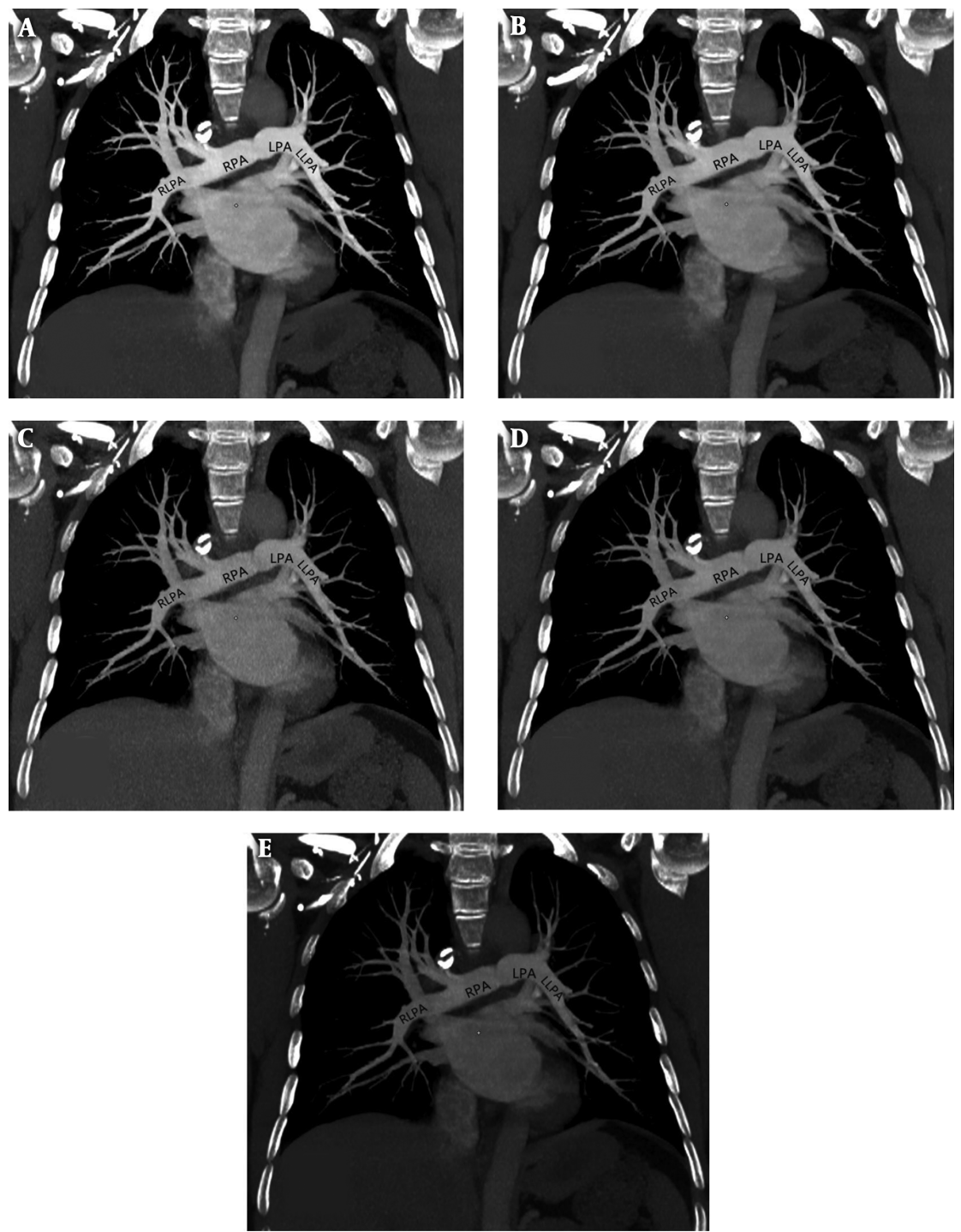

Figure 3. A 48-year-old female, body mass index $(\mathrm{BMI})=21.5 \mathrm{~kg} \cdot \mathrm{m}^{-2}$, figures A-E represent $60 \mathrm{keV}, 65 \mathrm{keV}, 70 \mathrm{keV}, 75 \mathrm{keV}$, and $80 \mathrm{keV}$ monochromatic images, respectively. A, 60 $\mathrm{keV}$ : SNR (mean) $=28.71, \mathrm{CNR}$ (mean) $=26.00, \mathrm{SD}$ (mean) $=19.62$, subjective score was 5 points; $\mathrm{B}, 65 \mathrm{keV}$ : SNR $($ mean $)=31.95, \mathrm{CNR}($ mean $)=28.77, \mathrm{SD}($ mean $)=14.32$, subjective score was 5 points; C, $70 \mathrm{keV}$ : $\mathrm{SNR}($ mean $)=11.98, \mathrm{CNR}($ mean $)=10.29, \mathrm{SD}($ mean $)=28.52$, subjective score was 4 points; D, $75 \mathrm{keV}: \mathrm{SNR}($ mean $)=19.53$, CNR $($ mean $)=15.89, \mathrm{SD}$ $($ mean $)=16.57$, subjective score was 4 points; $\mathrm{E}, 80 \mathrm{keV}$ : $\mathrm{SNR}($ mean $)=10.13, \mathrm{CNR}($ mean $)=7.14, \mathrm{SD}($ mean $)=15.98$, subjective score was 3 points.

our daily work.

CM passes through the superior vena cava into the right heart and then goes directly to the branches of the pulmonary artery from the right heart without systemic circulation in CTPA. Therefore, some authors believe that BMI has little effect on the use of CM dose in patients with CTPA. Our study also did not personalize CM dose based on patient's BMI. Our results indicated that there was no correlation between BMI with CNR and subjective scores of the $65 \mathrm{keV}$ monochromatic images, similar to the results of $\mathrm{Wu}$ et al. (20)

The ideal CTPA scan should collect data when the pulmonary artery is adequately enhanced, while the pulmonary vein is not enhanced, but the time window is very narrow, only 2 - 3 seconds. The bolus tracking (BT) technique commonly used at present is influenced by scanning experience, individual circulatory differences in patients and equipment. There is no uniform standard for the trigger threshold, monitoring point and start scan delay time, and it is more difficult to grasp the scan delay time when 

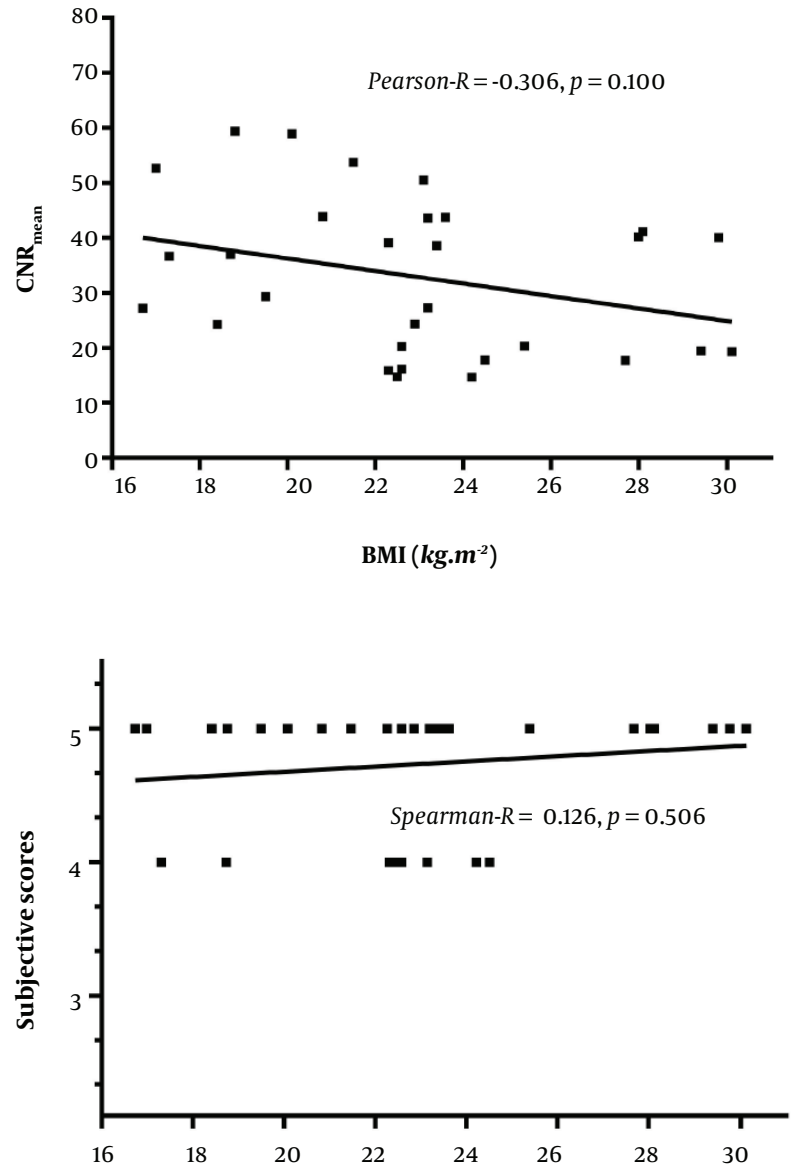

Figure 4. Scatter plot of correlation between body mass index (BMI) with contrastto-noise ratio (CNR) and subjective score of $65 \mathrm{keV}$ monochromatic images.

the dosage of contrast agent is low. In our study, a test bolus (TB) technique was used. The basic principle is to inject a small dose of contrast agent ( $8 \mathrm{~mL}$ in our study) before the formal scan. The time-density curve of pulmonary arterial enhancement was obtained, and the peak time of pulmonary arterial enhancement was obtained individually and accurately. Then a normal contrast dose $(25 \mathrm{~mL}$ in our study) for pulmonary artery imaging was injected to trigger the scanning at this time point to optimize pulmonary artery enhancement. The reason for adding 2 seconds to the peak enhancement time of TB in our study was that the peak enhancement time of contrast agent $(25 \mathrm{~mL})$ used for pulmonary artery imaging was usually delayed by 2 seconds. The advantage of TB was that it can reliably and accurately predict the peak time of pulmonary artery enhancement and eliminate the influence of individual differences, especially for patients with unstable hemody- namics $(36,37)$. The downside of TB was that additional small doses of CM and scanning were used before CTPA.

Some researchers compared the effects of the two monitoring methods on CTPA: Moradi and Khalili (38) showed that the two monitoring methods had no effect on the enhancement of pulmonary arteries in both groups $(\mathrm{P}>$ $0.05)$, while the incidence of artifacts in TB group was lower than that in BT group (11.5\% vs. $26.9 \%$ ), but there was no significant difference $(\mathrm{P}=0.159)$, and the results were consistent with the study carried out by Henzler et al. (39) and Johnson et al. (40). However, other studies have shown that pulmonary artery enhancement was higher in TB group (41). For example, Sucklings et al. (42) showed that pulmonary artery enhancement in the TB group was higher than that in the BT group (mean CT value was 396.24 $\pm 22.34 \mathrm{HU}$ vs. $329.33 \pm 20.92 \mathrm{HU}, \mathrm{P}<0.01$ ). However, in Suchling's study, the authors increased the injection rate of contrast agent from $4.0 \mathrm{~mL} / \mathrm{s}$ to $4.5 \mathrm{~mL} / \mathrm{s}$ in TB group, and the iodine flow rate increased accordingly, which may be the cause of higher enhancement in TB group. However, the standard deviation and range of CT value of pulmonary artery in TB group were larger, so the authors considered that the enhancement of pulmonary artery by BT technique was more uniform, which was similar to the results of in coronary CTA study performed by Cademartiri et al. (43) Rodrigues et al. (41) further analyzed the data of smaller patients with lower tube voltage $(100 \mathrm{kV})$ and found that there was no significant difference in pulmonary artery enhancement between the two monitoring methods. All the above studies were based on the conventional dosage of CM. Kerl et al. (44) compared two monitoring methods at low CM dosage $(50 \mathrm{~mL})$. The results showed that good pulmonary enhancement and high-quality images could be obtained in both TB and BT groups. There was no significant difference in pulmonary enhancement between the two groups $(\mathrm{P}>0.05)$. The reason for choosing TB in our study was to evaluate the peak enhancement of pulmonary artery more accurately.

There were limitations in this study. First, there was only one case of PE in the group. Second, the scanning parameters were not personalized according to BMI of each patient. Third, the lower monochromatic energy images were not reconstructed and the reason was due to the software limitation of our first generation dual-energy CT scanner that the images below $60 \mathrm{keV}$ (such as 40 - $60 \mathrm{keV}$ ) could not be reconstructed using ASiR algorithm on our CT system. Finally, even though iodine-based images in the dual-energy spectral CTPA can provide perfusion information in the lung parenchyma, this information was not analyzed since this study was aimed at exploring the feasibility 
of low CM volume in CTPA.

In conclusion, the $65 \mathrm{keV}$ monochromatic images provide the highest SNR, CNR and subjective score with the lowest image noise in the dual-energy spectral CT pulmonary angiography with low contrast dosage.

\section{Acknowledgments}

We would like to thank Dr. Jianying Li for editing the article.

\section{Footnotes}

Authors' Contributions: Study concept and design: Dong Han, Xiaoxia Chen, and Taiping He. Data acquisition: Xin Tian and Jing Chen. Analysis and interpretation of data: Dong Han and Yong Yu. Drafting of the manuscript: Dong Han. Critical revision of the manuscript for important intellectual content: Weihua Shi, Yong Yu, and Taiping He. Statistical analysis: Jieli Zhou and Mengting Liu.

Conflict of Interests: None declared.

Ethical Approval: This single-site prospective study was approved by our Institutional Review Board (the approval document no.: SZFYIEC-PF-2015 no. [08], Affiliated Hospital of Shaanxi University of Chinese Medicine, Xianyang).

Funding/Support: There was no financial assistance obtained from any organization or company for this manuscript.

Informed Consent: We obtained written informed consent signed by all patients before CTPA examination.

\section{References}

1. Konstantinides SV, Torbicki A, Agnelli G, Danchin N, Fitzmaurice D, Galie N, et al. Task force for the diagnosis and management of acute pulmonary embolism of the European Society of Cardiology (ESC). 2014 ESC guidelines on the diagnosis and management of acute pulmonary embolism. Eur Heart J. 2014;35(43):3033-69.3069a-3069k. doi: 10.1093/eurheartj/ehu283. [PubMed: 25173341].

2. Saade C, Mayat A, El-Merhi F. Exponentially decelerated contrast media injection rate combined with a novel patient-specific contrast formula reduces contrast volume administration and radiation dose during computed tomography pulmonary angiography. J Comput Assist Tomogr. 2016;40(3):370-4. doi: 10.1097/RCT.0000000000000371. [PubMed: 26854416].

3. Yuan R, Shuman WP, Earls JP, Hague CJ, Mumtaz HA, Scott-Moncrieff A, et al. Reduced iodine load at CT pulmonary angiography with dual-energy monochromatic imaging: comparison with standard CT pulmonary angiography-a prospective randomized trial. Radiology. 2012;262(1):290-7. doi: 10.1148/radiol.11110648. [PubMed: 22084206].

4. Bhalla S, Lopez-Costa I. MDCT of acute thrombotic and nonthrombotic pulmonary emboli. Eur J Radiol. 2007;64(1):54-64. doi:10.1016/j.ejrad.2007.06.032. [PubMed: 17686597].
5. Bosson JL, Pernod G, Joubin E, Hamidfar R, Bricault I, Hugon V, et al. Non-conform diagnostic management of pulmonary embolism suspected patients is responsible for a higher risk of thrombotic event occurrence. J Mal Vasc. 2007;32(1):15-22. doi:10.1016/j.jmv.2007.01.098. [PubMed: 17321710].

6. Wichmann JL, Katzberg RW, Litwin SE, Zwerner PL, De Cecco CN, Vogl TJ, et al. Contrast-induced nephropathy. Circulation. 2015;132(20):19316. doi: 10.1161/CIRCULATIONAHA.115.014672. [PubMed: 26572669].

7. Thomsen HS, Morcos SK. Contrast media and the kidney: European Society of Urogenital Radiology (ESUR) guidelines. Br J Radiol. 2003;76(908):513-8. doi: 10.1259/bjr/26964464. [PubMed: 12893691].

8. Nash K, Hafeez A, Hou S. Hospital-acquired renal insufficiency. Am J Kidney Dis. 2002;39(5):930-6. doi: 10.1053/ajkd.2002.32766. [PubMed: 11979336].

9. Marenzi G, Assanelli E, Campodonico J, Lauri G, Marana I, De Metrio $\mathrm{M}$, et al. Contrast volume during primary percutaneous coronary intervention and subsequent contrast-induced nephropathy and mortality. Ann Intern Med. 2009;150(3):170-7. doi: 10.7326/0003-4819-150-3200902030-00006. [PubMed: 19189906].

10. Nyman U, Bjork J, Aspelin P, Marenzi G. Contrast medium dose-to-GFR ratio: A measure of systemic exposure to predict contrast-induced nephropathy after percutaneous coronary intervention. Acta Radiol. 2008;49(6):658-67. doi: 10.1080/02841850802050762. [PubMed: 18568558].

11. Davenport MS, Khalatbari S, Cohan RH, Dillman JR, Myles JD, Ellis $\mathrm{JH}$. Contrast material-induced nephrotoxicity and intravenous lowosmolality iodinated contrast material: Risk stratification by using estimated glomerular filtration rate. Radiology. 2013;268(3):719-28. doi: 10.1148/radiol.13122276. [PubMed: 23579046].

12. Huda W, Ogden KM, Khorasani MR. Converting dose-length product to effective dose at CT. Radiology. 2008;248(3):995-1003. doi: 10.1148/radiol.2483071964. [PubMed: 18710988]. [PubMed Central: PMC2657852]

13. Hunsaker AR, Oliva IB, Cai T, Trotman-Dickenson B, Gill RR, Hatabu $\mathrm{H}$, et al. Contrast opacification using a reduced volume of iodinated contrast material and low peak kilovoltage in pulmonary CT angiography: Objective and subjective evaluation. AJR Am J Roentgenol. 2010;195(2):W118-24. doi: 10.2214/AJR.09.3342. [PubMed: 20651170].

14. Wittram C. How I do it: CT pulmonary angiography. $A J R A m$ Roentgenol. 2007;188(5):1255-61. doi: 10.2214/AJR.06.1104. [PubMed: 17449768].

15. Trad HS, Boasquevisque GS, Giacometti TR, Trad CY, Zoghbi Neto OS, Trad CS. Pulmonary 64-MDCT angiography with $50 \mathrm{~mL}$ of iodinated contrast material in an unselected patient population: A feasible protocol. Radiol Bras. 2016;49(2):75-8. doi: 10.1590/0100-3984.2014.0115. [PubMed: 27141128]. [PubMed Central: PMC4851474]

16. Mourits MM, Nijhof WH, van Leuken MH, Jager GJ, Rutten MJ. Reducing contrast medium volume and tube voltage in CT angiography of the pulmonary artery. Clin Radiol. 2016;71(6):615 e7-13. doi: 10.1016/j.crad.2016.03.005. [PubMed: 27059387].

17. Goble EW, Abdulkarim JA. CT pulmonary angiography using a reduced volume of high-concentration iodinated contrast medium and multiphasic injection to achieve dose reduction. Clin Radiol. 2014;69(1):36-40. doi: 10.1016/j.crad.2013.07.023. [PubMed: 24070908].

18. Faggioni L, Neri E, Sbragia P, Pascale R, D'Errico L, Caramella D, et al. 80-kV pulmonary CT angiography with $40 \mathrm{~mL}$ of iodinated contrast material in lean patients: Comparison of vascular enhancement with iodixanol (320 mg I/mL)and iomeprol (400 mg I/mL). AJR Am J Roentgenol. 2012;199(6):1220-5. doi: 10.2214/AJR.11.8122. [PubMed: 23169711]. 
19. Viteri-Ramirez G, Garcia-Lallana A, Simon-Yarza I, Broncano J, Ferreira $\mathrm{M}$, Pueyo JC, et al. Low radiation and low-contrast dose pulmonary CT angiography: Comparison of $80 \mathrm{kVp} / 60 \mathrm{ml}$ and $100 \mathrm{kVp} / 80 \mathrm{ml}$ protocols. Clin Radiol. 2012;67(9):833-9. doi: 10.1016/j.crad.2011.11.016. [PubMed: 22683247].

20. Wu CC, Lee EW, Suh RD, Levine BS, Barack BM. Pulmonary 64-MDCT angiography with $30 \mathrm{~mL}$ of IV contrast material: Vascular enhancement and image quality. AJR Am J Roentgenol. 2012;199(6):1247-51. doi: 10.2214/AJR.12.8739. [PubMed: 23169715].

21. Lu GM, Luo S, Meinel FG, McQuiston AD, Zhou CS, Kong X, et al. Highpitch computed tomography pulmonary angiography with iterative reconstruction at $80 \mathrm{kVp}$ and $20 \mathrm{~mL}$ contrast agent volume. Eur Radiol. 2014;24(12):3260-8. doi: 10.1007/s00330-014-3365-9. [PubMed: 25100336].

22. Carrascosa P, Leipsic JA, Capunay C, Deviggiano A, Vallejos J, Goldsmit A, et al. Monochromatic image reconstruction by dual energy imaging allows half iodine load computed tomography coronary angiography. EurJRadiol.2015;84(10):1915-20. doi:10.1016/j.ejrad.2015.06.019. [PubMed: 26152868].

23. Kulkarni NM, Sahani DV, Desai GS, Kalva SP. Indirect computed tomography venography of the lower extremities using single-source dual-energy computed tomography: Advantage of low-kiloelectron volt monochromatic images. J Vasc Interv Radiol. 2012;23(7):879-86. doi: 10.1016/j.jvir.2012.04.012. [PubMed: 22633619].

24. Lin XZ, Miao F, Li JY, Dong HP, Shen Y, Chen KM. High-definition CT gemstone spectral imaging of the brain: Initial results of selecting optimal monochromatic image for beam-hardening artifacts and image noise reduction. J Comput Assist Tomogr. 2011;35(2):294-7. doi: 10.1097/RCT.0b013e3182058d5c. [PubMed: 21412106].

25. Matsuda I, Akahane M, Sato J, Katsura M, Kiryu S, Yoshioka N, et al. Precision of the measurement of CT numbers: Comparison of dualenergy CT spectral imaging with fast $\mathrm{kVp}$ switching and conventional CT with phantoms.Jpn JRadiol. 2012;30(1):34-9. doi: 10.1007/s11604-0110004-0. [PubMed: 22135112].

26. Matsumoto K, Jinzaki M, Tanami Y, Ueno A, Yamada M, Kuribayashi S. Virtual monochromatic spectral imaging with fast kilovoltage switching: Improved image quality as compared with that obtained with conventional 120-kVp CT. Radiology. 2011;259(1):257-62. doi: 10.1148/radiol.11100978. [PubMed: 21330561].

27. Cheng J, Yin Y, Wu H, Zhang Q, Hua J, Hua X, et al. Optimal monochromatic energy levels in spectral CT pulmonary angiography for the evaluation of pulmonary embolism. PLoS One. 2013;8(5). e63140. doi: 10.1371/journal.pone.0063140. [PubMed: 23667583]. [PubMed Central: PMC3646731].

28. Apfaltrer P, Sudarski S, Schneider D, Nance JJ, Haubenreisser H, Fink $\mathrm{C}$, et al. Value of monoenergetic low-kV dual energy CT datasets for improved image quality of CT pulmonary angiography. Eur J Radiol. 2014;83(2):322-8. doi: 10.1016/j.ejrad.2013.11.005. [PubMed: 24361061].

29. Ohana M, Labani A, Jeung MY, El Ghannudi S, Gaertner S, Roy C. Iterative reconstruction in single source dual-energy CT pulmonary angiography: Is it sufficient to achieve a radiation dose as low as stateof-the-art single-energy CTPA? Eur J Radiol. 2015;84(11):2314-20. doi: 10.1016/j.ejrad.2015.07.010. [PubMed: 26210093].

30. Saade C, Bourne R, El-Merhi F, Somanathan A, Chakraborty D, Brennan P. An optimised patient-specific approach to administration of contrast agent for CT pulmonary angiography. Eur Radiol. 2013;23(11):3205-12. doi: 10.1007/s00330-013-2919-6. [PubMed: 23732690].

31. Piechowiak EI, Peter JF, Kleb B, Klose KJ, Heverhagen JT. Intravenous iodinated contrast agents amplify DNA radiation damage at CT. Radiology. 2015;275(3):692-7. doi: 10.1148/radiol.14132478. [PubMed:
25654667].

32. Wang F, Zhang Y, Xue H, Han W, Yang X, Jin Z, et al. Combined use of iterative reconstruction and monochromatic imaging in spinal fusion CT images. Acta Radiol. 2017;58(1):62-9. doi: 10.1177/0284185116631182. [PubMed: 26917784].

33. Zhu Z, Zhao XM, Zhao YF, Wang XY, Zhou CW. Feasibility study of using gemstone spectral imaging (GSI) and adaptive statistical iterative reconstruction (ASIR) for reducing radiation and iodine contrast dose in abdominal CT patients with high BMI values. PLoS One. 2015;10(6). e0129201. doi: 10.1371/journal.pone.0129201. [PubMed: 26079259]. [PubMed Central: PMC4469609].

34. Ma CL, Chen XX, Lei YX, Zhang XR, Jia YJ, Tian X, et al. Clinical value of dual-energy spectral imaging with adaptive statistical iterative reconstruction for reducing contrast medium dose in CT portal venography: in comparison with standard $120-\mathrm{kVp}$ imaging protocol. $\mathrm{Br}$ J Radiol. 2016;89(1062):20151022. doi: 10.1259/bjr.20151022. [PubMed: 27031376]. [PubMed Central: PMC5258165].

35. Zhao L, Winklhofer S, Yang Z, Wang K, He W. Optimal adaptive statistical iterative reconstruction percentage in dual-energy monochromatic CT portal venography. Acad Radiol. 2016;23(3):337-43. doi: 10.1016/j.acra.2015.11.004. [PubMed: 26777591].

36. Bae KT. Intravenous contrast medium administration and scan timing at CT: Considerations and approaches. Radiology. 2010;256(1):3261. doi: 10.1148/radiol.10090908. [PubMed: 20574084].

37. Fleischmann D. CT angiography: Injection and acquisition technique. Radiol Clin North Am. 2010;48(2):237-47. vii. doi: 10.1016/j.rcl.2010.02.002. [PubMed: 20609872].

38. Moradi M, Khalili B. Qualitative indices and enhancement rate of CT pulmonary angiography in patients with suspected pulmonary embolism: Comparison between test bolus and bolus-tracking methods. Adv Biomed Res. 2016;5:113. doi: 10.4103/2277-9175.184309. [PubMed: 27403408]. [PubMed Central: PMC4926546].

39. Henzler T, Meyer M, Reichert M, Krissak R, Nance JJ, Haneder S, et al Dual-energy CT angiography of the lungs: Comparison of test bolus and bolus tracking techniques for the determination of scan delay. Eur J Radiol. 2012;81(1):132-8. doi: 10.1016/j.ejrad.2010.06.023. [PubMed: 20621430].

40. Johnson TR, Nikolaou K, Wintersperger BJ, Knez A, Boekstegers P, Reiser MF, et al. ECG-gated 64-MDCT angiography in the differential diagnosis of acute chest pain. AJR Am J Roentgenol. 2007;188(1):76-82. doi: 10.2214/AJR.05.1153. [PubMed:17179348].

41. Rodrigues JC, Mathias H, Negus IS, Manghat NE, Hamilton MC. Intravenous contrast medium administration at 128 multidetector row CT pulmonary angiography: Bolus tracking versus test bolus and the implications for diagnostic quality and effective dose. Clin Radiol. 2012;67(11):1053-60. doi: 10.1016/j.crad.2012.02.010. [PubMed: 22520034].

42. Suckling T, Smith T, Reed W. A retrospective comparison of smart prep and test bolus multi-detector CT pulmonary angiography protocols. J Med Radiat Sci. 2013;60(2):53-7. doi: 10.1002/jmrs.17. [PubMed: 26229608]. [PubMed Central: PMC4175799].

43. Cademartiri F, Nieman K, van der Lugt A, Raaijmakers RH, Mollet N, Pattynama PM, et al. Intravenous contrast material administration at 16-detector row helical CT coronary angiography: Test bolus versus bolus-tracking technique. Radiology. 2004;233(3):817-23. doi: 10.1148/radiol.2333030668. [PubMed: 15516601].

44. Kerl JM, Lehnert T, Schell B, Bodelle B, Beeres M, Jacobi V, et al. Intravenous contrast material administration at high-pitch dual-source CT pulmonary angiography: Test bolus versus bolus-tracking technique. EurJ Radiol. 2012;81(10):2887-91. doi: 10.1016/j.ejrad.2011.09.018. [PubMed: 22019597]. 